\title{
Effect of Microstructure on High-Temperature Mechanical Behavior of Nickel-Base Superalloys for Turbine Disc Applications
}

\author{
Heather J. Sharpe ${ }^{1, a}$ and Ashok Saxena ${ }^{2, b}$ \\ ${ }^{1}$ Rolls-Royce Corporation, $2001 \mathrm{~S}$. Tibbs Avenue, Indianapolis, IN 46241 USA \\ ${ }^{2}$ University of Arkansas, 4183 Bell Engineering Center, Fayetteville, AR 72701 USA \\ aheather.j.sharpe@rolls-royce.com, basaxena@uark.edu
}

Key words: PM superalloys, Nickel-base, microstructure, mechanical properties

\begin{abstract}
The objective of this work was to establish relationships between alloy microstructure and high temperature mechanical properties such as strength, creep, and creep crack growth in Nickel-base powder metallurgy superalloys. Systematic variations of super solvus heat-treatments generated test material from three next-generation turbine disc alloys. Quantification of key microstructural features such as $\gamma^{\prime}$ distribution and morphology and grain boundary serrations was coupled with mechanical testing results in order to optimize microstructure for operating conditions specific to the bore region.
\end{abstract}

\section{Introduction}

By improving the temperature capabilities of nickel-base superalloys, the traditional choice for use in key components of gas turbines such as discs and blades, the increasing demands for higher efficiencies in the aviation and power generation fields can be met. It is suggested that alloys used in these applications must improve their current temperature capabilities from about $650^{\circ} \mathrm{C}$ to approximately $700^{\circ} \mathrm{C}$. Tailoring of alloy microstructures to optimize high temperature performance offers an economical option for achieving these improvements.

\section{Materials}

Three nickel-base superalloys were considered in this study: LSHR, Alloy10, and RR1000. All of these powder metallurgy alloys are under consideration for use as turbine disc material in the next generation of gas turbine engines.

Ni-base Superalloys. All materials were produced via traditional powder metallurgy routes including producing powder by gas atomization, hot compaction and extrusion and isothermal forging. All materials were produced by Special Metals Corp. and forged by Ladish Co. LSHR and Alloy10 were produced for NASA Glenn Research Center and RR1000 was produced for RollsRoyce plc.

Table 1 Nominal Composition of featured alloys by weight percent. $\gamma$ ' solvus temperature for each is included.

\begin{tabular}{|c|c|c|c|c|c|c|c|c|c|c|c|c|c|c|}
\cline { 2 - 16 } \multicolumn{1}{c|}{} & Ni & Al & B & C & Co & Cr & Hf & Mo & Nb & Ta & Ti & W & Zr & y' Solvus \\
\hline LSHR & Bal. & 3.5 & 0.03 & 0.03 & 20.7 & 12.5 & 0 & 2.7 & 1.5 & 1.6 & 3.5 & 4.3 & 0.05 & $1160^{\circ} \mathrm{C}$ \\
\hline Alloy 10 & Bal. & 3.69 & 0.03 & 0.03 & 14.9 & 10.2 & 0 & 2.73 & 1.87 & 0.9 & 3.93 & 6.2 & 0.1 & $1182^{\circ} \mathrm{C}$ \\
\hline RR1000 & Bal. & 3 & 0.02 & 0.03 & 18.5 & 15 & 0.75 & 5 & 0 & 2 & 3.6 & 0 & 0.06 & $1150^{\circ} \mathrm{C}$ \\
\hline U720 & Bal. & 2.5 & 0.035 & 0.035 & 15 & 18 & 0 & 3 & 0 & 0 & 5 & 1.25 & 0.035 & $1190^{\circ} \mathrm{C}$ \\
\hline
\end{tabular}

These alloys represent various advances in mechanical properties and ease of production or machining based on changes in chemistry or microstructure from previous generations of superalloys. Their chemical compositions, seen in Table 1, have many similarities, with the main differences being in relative amounts of hafnium, molybdenum, niobium, tantalum, and tungsten. At the time of publication none of these allows had seen use in production hardware, although development work is rapidly progressing. U720 is listed for comparison to a currently used alloy for turbine rotor applications. 
Heat-Treatment. Supersolvus heat treat conditions were chosen to focus the work on coarse grain microstructures optimized for the demanding operating conditions that may be seen in the bore of a turbine rotor. Solution temperatures were chosen specific to the $\gamma^{\prime}$ solvus temperature of each alloy; cooling rates were chosen to be representative of those achievable in a production process.

A matrix of 54 alloy-microstructure combinations were produced for hot hardness and hot tensile testing, as well as the microstructural quantification work. These 54 microstructures represent combinations of the three Ni-base disc alloys, three supersolvus solutioning temperatures, three cooling rates, and two secondary holds. Additional LSHR material was produced for creep deformation, and creep and fatigue crack growth tests. Hold temperatures and times were held consistent with previous NASA studies [1], with cooling rate from supersolvus solution heat treatment being the only variable.

Microstructures. Several relevant microstructural features were imaged and quantified for consideration in relation to mechanical behavior. The techniques used for sample preparation and imaging varied based on the feature of interest. Techniques that could be applied on all three materials were sought in order to maximize consistency. ASTM standards were used for quantification of the microstructure whenever possible, although standards were not available for the quantification of some features. Coupons of material were taken from tensile bar blanks so as to assure consistency between tested material and documented microstructures.

Grain size for as-received PM material had a mean diameter of approximately $10-15 \mu \mathrm{m}$ or ASTM 11-12. Following supersolvus heat treatment, grain sizes were tightly clustered between 24 and $54 \mu \mathrm{m}$ for all materials. RR1000 produced the smallest grains for all treatments while Alloy 10 produced the largest. Average grain diameters for LSHR, Alloy10, and RR1000 were $41.8 \mu \mathrm{m}$ (ASTM 5.9), $44.7 \mu \mathrm{m}$ (ASTM 5.7), and $33.3 \mu \mathrm{m}$ (ASTM 6.5), respectively. Consistent with expectations, grain size variations were most strongly tied to the solution temperature.

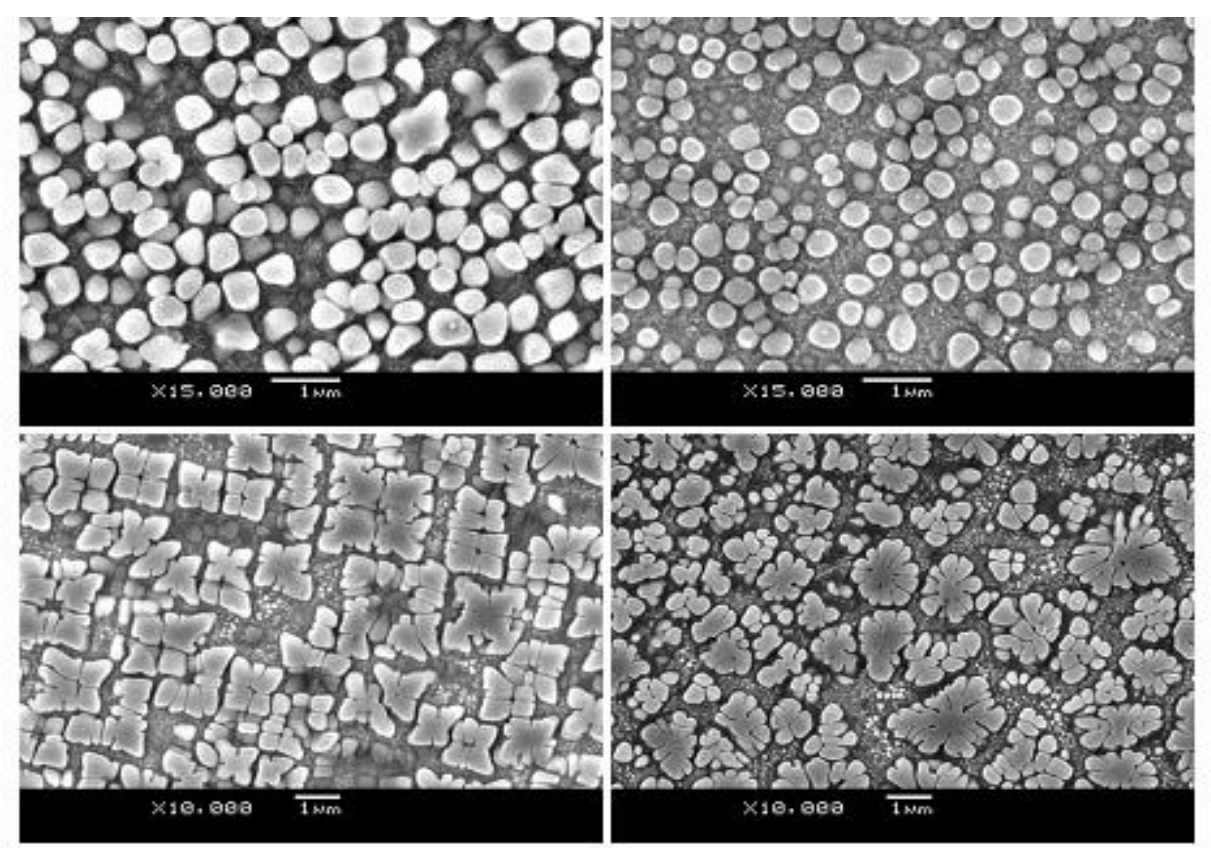

Figure 1 Representative micrographs showing variation of size and morphology with material and cooling rate. $\gamma^{\prime}$ is seen in relief in all images with fine tertiary $\gamma^{\prime}$ visible in channels between secondary (cooling) $\gamma^{\prime}$. Fast cooled LSHR (top left), fast cooled RR1000 (top right) slow cooled LSHR (bottom left) versus slow cooled RR1000 (bottom right) micrographs are shown.

Secondary (cooling) $\gamma^{\prime}$ precipitate size and size distributions for each specimen were generated based on measurements of 100-300 individual precipitate particles to validate the use of an average size. The majority of specimens displayed a basically normal size distribution with some skewing to the right; all were mono-modal distributions. Precipitate size was most directly correlated to cooling rate, with no apparent relationship to solution temperature or secondary hold temperature. 
Morphology of secondary $\gamma^{\prime}$ precipitates was considered in the form of circularity measurements. Initially, all precipitates were spherical, forming into alternative shapes as the particle grew with cooling and ageing. LSHR and Alloy10 formed distinctly cuboidal precipitates (seen as square or diamond in cross-section). Both show a distinct tendency to form a pseudodendritic structure by preferential growth on the corners of the cubic precipitates. The RR1000 material, on the other hand, shows little indication of cuboidal or solid-state dendritic formation. Instead, the spherical particles seem to form a still spherical lobed structure. Again, cooling rate was the most directly correlated heat treatment variable to affect the quantified feature.

Grain boundary serrations were characterized in detail. Grain boundary curvature ratio (GBCR) was characterized for each specimen to capture the extent of serrations, while an average wavelength and amplitude for the serrated boundaries were calculated to capture the nature of the serrations. GBCR is the ratio of actual length versus shortest distance between end points. Similar values for GBCR were measured for each material. All values for GBCR fell between 1.08 (least serrated) to 1.74 (most serrated), with a value of 1 indicating no serrations.

Photos in Figure 2 represent the amount of variation achieved from iterations of supersolvus solution temperature, cooling rate, and hold temperature. More detailed discussion of processingmicrostructure relationships is beyond the scope of this paper but can be reviewed elsewhere [2]. Visual and quantitative results confirm the direct and nuanced control that can be exercised over microstructure through heat treatment. This can then be translated into control over mechanical behavior as in seen the following discussion.

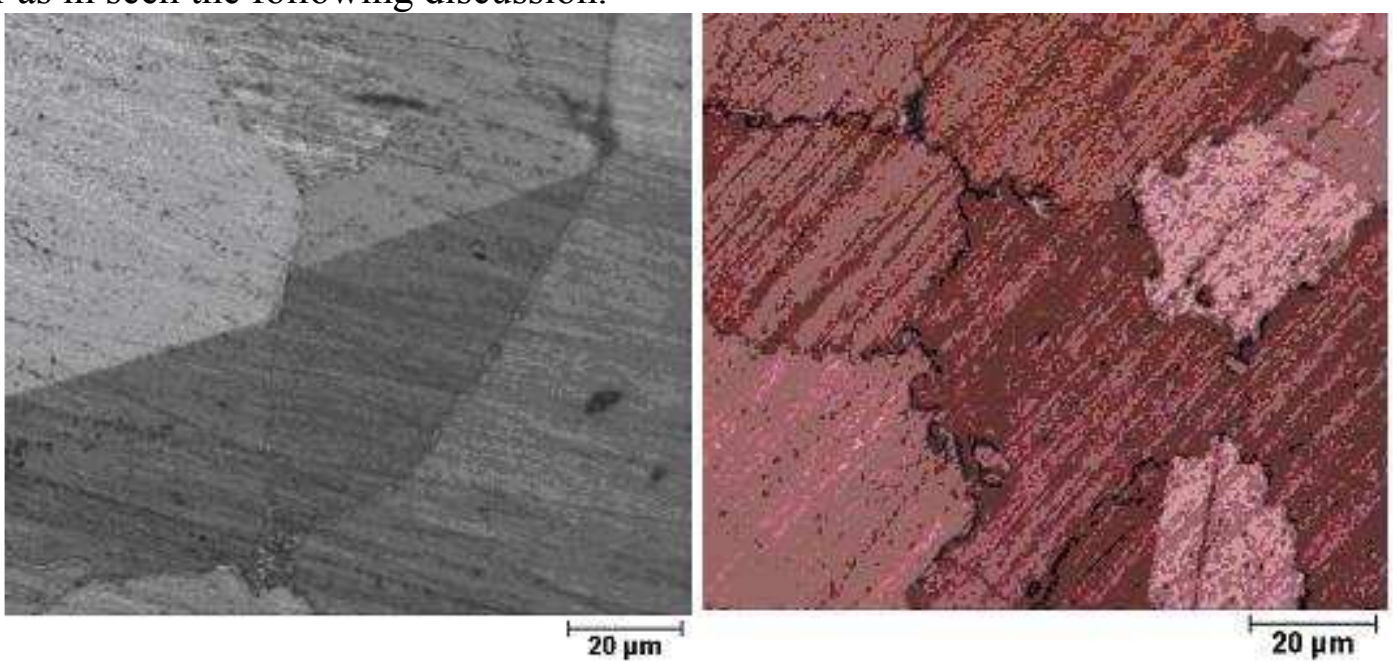

Figure 2 Example of straight and serrated grain boundaries (LSHR and Alloy10 respectively).

\section{Mechanical Behavior}

High temperature mechanical testing is conducted to better understand the relationship between microstructure and mechanical properties. This serves to optimize the performance of the materials through control of processing variables. Samples from each variant of heat treatment were used in high temperature tensile tests to explore the interaction between microstructure and strength. Subsequent testing focused on the effect microstructure on damage tolerance through creep deformation and creep crack growth testing of LSHR material.

High Temperature Tensile Behavior. Innumerable studies of superalloys have established the superior strength achieved with fine-grain materials, while coarse-grain is optimal for creep resistance. Given that fine grain material is best for strength; these tests concentrated on establishing how to optimize strength within the limitations of a coarse-grain material. Results from tensile tests clarified applicable strengthening mechanisms by establishing correlations between microstructural features and strength.

Tensile tests were conducted on the full set of 54 specimens at $704^{\circ} \mathrm{C}$ in air in accordance with ASTM E8 [3]. Of the three alloys tested LSHR showed the highest values for ultimate tensile strength (UTS) and yield strength (YS). Although the difference in UTS results between alloys was greater than the difference in YS, this trend was consistent for both. 
Table 2 Summary of tensile testing results at $704 \mathrm{C}$.

\begin{tabular}{|c|c|c|c|}
\cline { 3 - 4 } \multicolumn{2}{c|}{} & \multicolumn{1}{c|}{$\begin{array}{c}\text { Yield Strength } \\
(0.2 \% \text { offset })[\mathrm{MPa}]\end{array}$} & $\begin{array}{c}\text { Ultimate Tensile } \\
\text { Strength [MPa }]\end{array}$ \\
\hline \multirow{2}{*}{ Alloy } & Highest & 958.3 & 1278.2 \\
\cline { 2 - 4 } & Lowest & 787.3 & 1148.5 \\
\hline \multirow{2}{*}{ Alloy10 } & Highest & 911.4 & 1202.3 \\
\cline { 2 - 4 } & Lowest & 754.2 & 1080.9 \\
\hline \multirow{2}{*}{ RR1000 } & Highest & 857.6 & 1215.4 \\
\cline { 2 - 4 } & Lowest & 721.1 & 1045.8 \\
\hline
\end{tabular}

No apparent correlation to solution temperature (and therefore grain size) was observed over the range investigated. Beyond variation with composition, results for high temperature strength were most closely linked to the cooling rate and a weaker correlation to hold temperature. Overall, specimens cooled at the slowest rate produced the lowest values of UTS and yield strength. Intermediate and fast cooling rates produced similar results to each other. Also, lower UTS and YS values resulted from materials subjected to the $845^{\circ} \mathrm{C}$ hold, as opposed to the $1040^{\circ} \mathrm{C}$ hold. The effect of secondary hold on the strength seemed to be more pronounced within the slow cooled specimens.

As with most high temperature mechanical properties, especially in highly complex material systems, multiple mechanisms tend to operate simultaneously. This certainly seems to be the case for high temperature strength, where results trend with both composition and precipitate size and distribution, suggesting the role of solid solution strengthening and precipitate strengthening respectively.

Results for hot tensile tests clearly showed the superior high temperature tensile strength of LSHR. Testing conducted at NASA-Glenn Research Center corroborated the superior strength of LSHR. Results from this work claimed strength values that "exceeded those of ME3 and Udimet720" [4]. With the highest content of matrix-solute elements, LSHR saw the biggest improvement from solid solution strengthening. The role of precipitate strengthening was seen in all materials by the maximization of strength through minimization of precipitate size and spacing which was achieved by fast cooling temperatures and higher hold temperatures.

Creep Deformation. Understanding the creep deformation behavior of Ni-base superalloys is crucial since these materials are intended for high temperature applications. As mentioned previously, coarse-grain microstructures have been proven to provide superior creep resistance. The creep tests conducted for this work sought to establish what further refinements could be made to the microstructure of supersolvus coarse grain material to improve creep resistance.

Creep deformation tests were carried out on LSHR material for three different microstructures at various stress levels. All tests were carried out at $704{ }^{\circ} \mathrm{C}$ in air in accordance with A STM E139 [5] and allowed to continue to failure. The variations in microstructure investigated in this section were only those related to cooling rate, while all other processing parameters were held constant. Thus, the three microstructures tested had the same grain size, but different $\gamma^{\prime}$ sizes and some variation in grain boundary serration formations. The cooling rates considered here were much more rapid than those in the matrix of microstructures used for tensile testing in order to be more consistent with those produced by industry procedures. However, quantified results for all microstructures trend together well.

Test results for time to 1\% creep and time to failure as a function of cooling rate are presented in Figure 3 and Figure 4 respectively. For all of the creep tests, a faster cooling rate (i.e. smaller $\gamma^{\prime}$ precipitates) produced longer times to a given percent creep or failure. This suggests that a fast cooling rate produces a microstructure optimized for creep deformation resistance. A consideration of the creep mechanisms active in this regime is necessary to justify why small $\gamma^{\prime}$ precipitates are optimal.

During creep deformation processes, multiple mechanisms can be taking place concurrently or sequentially. While multiple mechanisms are operating at the same time, some are more relevant than others given the microstructure in question. Because all specimens are of a consistent, coarse grain size, grain boundary density is low and constant and therefore creep mechanisms such as grain boundary sliding facilitated by Nabarro-Herring creep and Coble creep can be de-emphasized. 


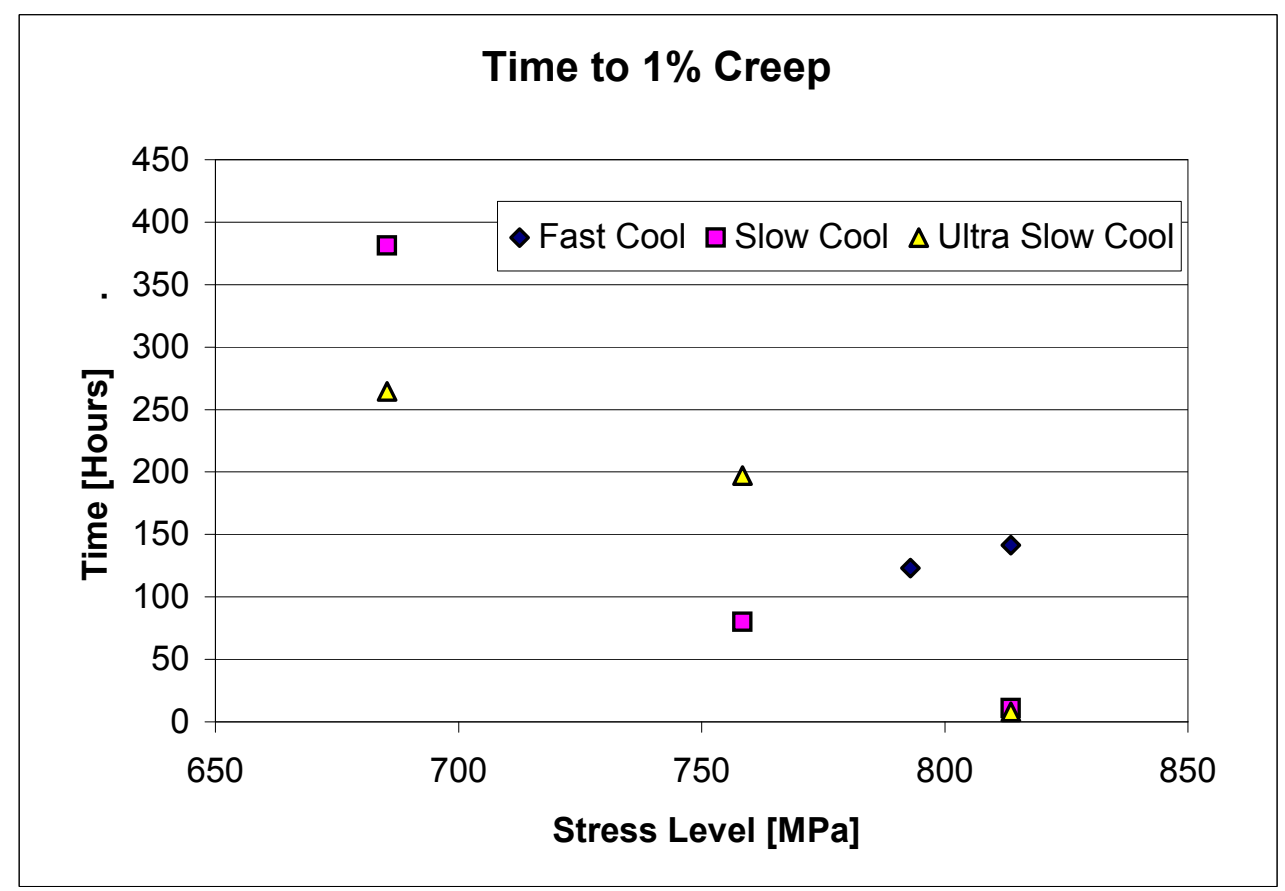

Figure 3 Time to $1 \%$ creep as a function of stress level for three microstructures of LSHR.

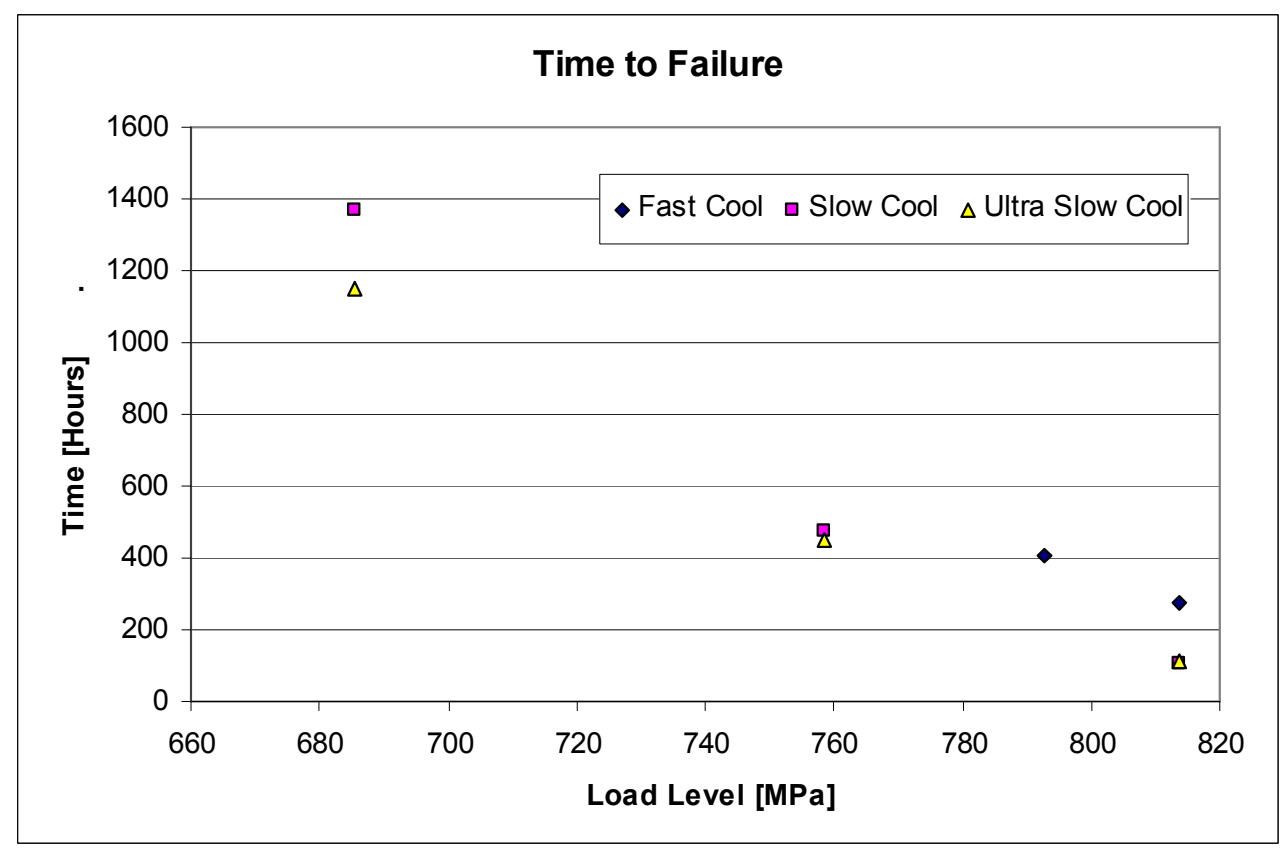

Figure 4 Time to failure as a function of stress level for three microstructures of LSHR.

Although diffusional flow still occurs, dislocation creep is the primary creep mechanism affected by variations in $\gamma^{\prime}$ size and morphology. It is proposed that $\gamma^{\prime}$ precipitates act as obstacles to dislocation motion within the context of dislocation creep. Creep test results showed that specimens with a more rapid cooling rate produced the slowest creep rates and longest times to failure. Rapid cooling rates produce a fine dispersion of precipitate particles. Investigation of fracture surface confirm an intergranular creep failure mode typical for conditions of limited grain boundary sliding leading to the coalescence of crack at the grain boundaries. It is interesting to note that the microstructural feature of grain boundary serrations, which had been quantified in two dimensions, can be observed as a three dimensional surface roughness on intergranular fracture surfaces. 
Results from creep deformation testing of variations of coarse grain LSHR suggest that rapid cooling of specimens improves the creep capabilities of the alloy at $704^{\circ} \mathrm{C}$. Creep strain rates were minimized and time to $1 \%$ creep and time to failure were extended. Grain size remained constant for each specimen tested, so improvements to creep must be a function of mechanisms internal to the grain. This is attributed to the role of $\gamma^{\prime}$ particles as obstacles to dislocation motion within the context of dislocation creep.

Creep Crack Growth. Limited creep crack growth testing was conducted on slow and fast cooled coarse grain LSHR specimens in air at $704^{\circ} \mathrm{C}$ in accordance with ASTM E1457 [6]. Results from creep crack growth testing indicate a significant difference in crack growth rates for specimens of different cooling rates. Crack growth rates for slow cooled specimens were an order of magnitude slower than were the fast cooled specimens. Early results suggest that improved creep crack growth resistance can be achieved with a microstructure that includes coarser precipitate particles and more highly serrated grain boundaries.

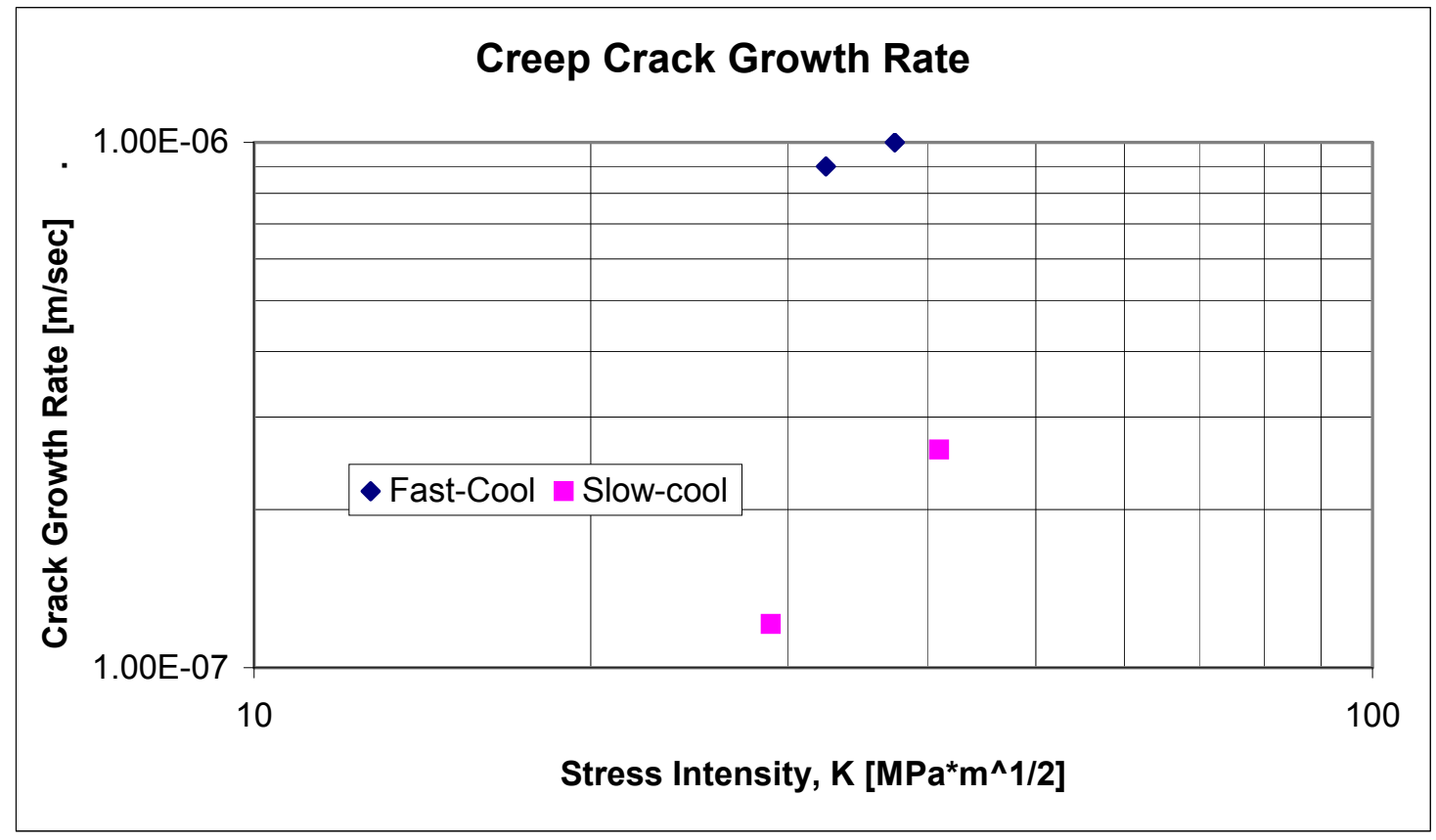

Figure 5 Graph of estimated crack growth rates for creep crack growth tests.

\section{Conclusions and Future Work}

This work demonstrates the direct correlation between alloy microstructure and high temperature mechanical behavior for next-generation PM Nickel-base superalloys. Control of microstructure facilitates the efficient improvement alloy performance for a given application. Future work should extend the results of this work into the field of computer learning in order to further the development of applications such as dual-microstructure discs. Initial efforts associated with this work demonstrate the potential contribution of artificial neural networks to the advancement of high temperature alloy performance.

\section{References}

[1] T. Gabb, J. Gayda, J. Falsey, "Forging of Advanced Disk Alloy LSHR". NASA T/M 2005213649, 2005.

[2] H. Sharpe. Effect of Microstructure on High-Temperature Mechanical Behavior of Nickel-Base Superalloys for Turbine Disc Applications. Diss. Georgia Institute of Technology, 2007.

[3] ASTM Standard E8, ASTM International, 2004.

[4] T. Gabb, J. Gayda, J. Telesman, P. Kantzos,"Thermal and Mechanical Property Characterization of the Advanced Disk Alloy LSHR”. NASA T/M 2005-213645, 2005.

[5] ASTM Standard E139-00, ASTM International, 2000.

[6] ASTM Standard E1457, ASTM International, 2000. 